\title{
Efecto de una estrategia pedagógica sobre el desarrollo metacognitivo de adolescentes chilenos
}

\author{
A pedagogical strategy effect on metacognitive development of Chilean adolescents
}

\author{
Sonia Eliana Osses Bustingorry ${ }^{a}$, Laura Eliana Carrasco Delgado, \\ José Luis Gálvez. Nieto ${ }^{c}$ \\ ${ }^{a}$ Universidad de La Frontera. \\ Correo electrónico: sonia.osses@ufrontera.cl \\ ${ }^{\mathrm{b}}$ Universidad de La Frontera. \\ Correo electrónico: laura.carrasco@ufrontera.cl. \\ ${ }^{\mathrm{c}}$ Universidad de La Frontera. \\ Correo electrónico: jose.galvez@ufrontera.cl.
}

\begin{abstract}
RESUMEN
En este artículo, basado en una investigación mixta, se presentan los avances generados en el desarrollo metacognitivo de estudiantes chilenos, a partir de una intervención pedagógica en Biología. En el ámbito cuantitativo - cuyos resultados incluye el artículo - se utilizó un diseño cuasi experimental que consistió en evaluación inicial de la metacognición en los estudiantes (pretest), intervención pedagógica, y evaluación final (postest). El desarrollo de la metacognición correspondió a la diferencia entre las mediciones final e inicial.

Los resultados obtenidos mostraron que, de las seis dimensiones metacognitivas analizadas, cinco presentaron diferencias significativas. La evaluación de su magnitud indicó que las cinco dimensiones señaladas mostraron una diferencia grande, según la escala de Morales (Morales 2008). Conocimiento, presenta la mayor distancia entre la medición pre-test y post-test $(\mathrm{d}=2.45)$, le sigue la dimensión Evaluación $(\mathrm{d}=2.03)$, luego Planificación $(\mathrm{d}=1.63)$, Estrategias $(\mathrm{d}=1.04) \mathrm{y}$, finalmente, Control-Supervisión $(\mathrm{d}=.62)$.
\end{abstract}

Palabras clave: intervención pedagógica, estudiantes chilenos, dimensiones metacognitivas, sistema educacional.

\section{ABSTRACT}

This article, based on mixed research, presents the advances generated in the metacognitive development of Chilean students from a pedagogical intervention in Biology. In the quantitative field - the results being included in the article - we used a quasi-experimental design that consisted of an initial evaluation of the students' metacognition (pre-test); a pedagogical intervention, the final evaluation (post-test). The development of metacognition was determined from the difference between the final and initial measurements.

The results obtained showed that, of the six metacognitive dimensions analyzed, five presented significant differences after the pedagogical intervention. The evaluation of its magnitude indicated that the five dimensions mentioned above showed a large difference, according to the scale of Morales (Morales 2008). Knowledge presents the greatest distance between the pre-test and post-test measurement $(\mathrm{d}=2.45)$, then the Evaluation dimension $(\mathrm{d}=2.03)$, next Planning $(\mathrm{d}=1.63)$, Strategies $(\mathrm{d}=1.04)$ and finally, Control-Supervision $(\mathrm{d}=.62)$.

Keywords: pedagogical intervention, Chilean students, metacognitive dimensions, educational system. 


\section{INTRODUCCIÓN}

\subsection{CONCEPTO DE METACOGNICIÓN}

La metacognición aplicada a la educación, es un constructo que ha experimentado gran desarrollo en las últimas décadas a nivel nacional e internacional (Peronard 2009; Paz 2011; Jerónimo y Ayala 2011).

Uno de los primeros autores que se refiere a la metacognición, la define como "el conocimiento que uno tiene acerca de los propios procesos y productos cognitivos, o cualquier otro asunto relacionado con ellos" (Flavell 1976: 232). Complementando esta definición, Brown y Smiley señalan que el dominio de la metacognición se extiende desde el conocimiento que el sujeto tiene de sus procesos cognitivos hasta el control de su propia actividad cognitiva (Brown y Smiley 1978).

Según Mateos, el conocimiento metacognitivo incluye el conocimiento de la persona y sus capacidades, de la tarea y de las estrategias de aprendizaje. Tal conocimiento empieza a manifestarse durante los años de educación básica y logra un desarrollo más o menos completo alrededor de los 11 a 12 años y en la adolescencia (Mateos 2002). En esta línea, Soto afirma que los procesos de control metacognitivo: planificación, supervisión y evaluación del propio conocimiento y de la propia actividad cognitiva, se ponen en marcha antes, durante y después de la ejecución de la tarea. Se trata de procesos referidos a un plano reflexivo, que va más allá de lo cognitivo, de modo tal, que los estudiantes menos competentes tenderían a ir directamente a cualquier acción, sin planificarla previamente y sin supervisar su ejecución (Soto 2002).

Profundizando en el concepto de metacognición, se puede expresar que:

Se practica la metacognición cuando se tiene conciencia de la mayor dificultad para aprender un tema respecto de otro, cuando se comprende que se debe verificar un fenómeno antes de aceptarlo como un hecho, cuando se piensa que es preciso examinar todas y cada una de las alternativas antes de decidir cuál es la mejor, cuando se advierte que se debería tomar nota de algo, porque puede olvidarse (Osses y Jaramillo 2008).

\subsection{METACOGNICIÓN Y PROCESO DE ENSEÑANZA Y APRENDIZAJE, CON ÉNFASIS EN CIENCIAS NATURALES}

En relación con los procesos necesarios para resolver problemas en clases de Química General en España, Solaz plantea estrategias didácticas que permitan mejorar significativamente el rendimiento de los alumnos, tales como: comprensión y asimilación conceptual del tema (por ejemplo uso de mapa conceptual); reemplazo de los métodos de enseñanza tradicional por prácticas instruccionales para desarrollar capacidades básicas (comprensión lectora, control de la propia comprensión) y de razonamiento científico (explicar, interpretar, razonar críticamente, tomar decisiones); utilización de modelos, dibujos, diagramas, símbolos; intercambio de ideas y opiniones mediante el trabajo grupal; elaboración de modelos mentales y actividades de carácter metacognitivo, como parte del currículo científico (Solaz 2010).

Analizando hábitos y estrategias de estudio en alumnos de Química Inorgánica en una Universidad de Argentina, De Bullaude, Córdoba, Torres, y De Morán, a través de encuestas 
y cuestionarios, observaron que los alumnos no utilizaban metodologías de trabajo intelectual en el estudio, debido al uso de inadecuadas metodologías de enseñanza -aprendizaje en los años de estudio previo. Señalan que es necesario incorporar metodologías educacionales que favorezcan la adquisición de hábitos eficientes de estudio que conduzcan a los estudiantes a practicar la metacognición y a fijar objetivos de aprendizaje que les permitan la adquisición, ampliación y aplicación de la información (De Bullaude, Córdoba, Torres, y De Morán 2008).

Jerónimo y Ayala, se refieren a la importancia de la relación pedagógica, en el proceso de enseñanza-aprendizaje de las Ciencias Naturales, entre el profesor y 25 estudiantes de Biología Molecular del programa de salud de la Universidad Autónoma de Manizales, Colombia, concluyendo que la relación pedagógica logra importancia al transformarse en un agente de cambio en la forma de enseñar y aprender, cuando se incluye la cognición, metacognición y motivación (Jerónimo y Ayala 2011).

Pérez de Albérniz, Escolano, Pascual, Lucas y Sastre, señalan que en estudios realizados en universidades europeas, se ha llegado a la conclusión que el uso de la metacognición en el proceso del aprendizaje, facilita a los alumnos un mayor dominio de los contenidos estudiados, mediante la elaboración autónoma y cooperativa, en comparación con otras técnicas de estudio que más bien se basan en el aprendizaje receptivo (Pérez de Albérniz, Escolano, Pascual, Lucas y Sastre 2013).

Para Tesouro, el trabajo de las habilidades metacognitivas debe ser permanente en el tiempo, de modo que los estudiantes aprendan a pensar y, de esa manera lograr un aprendizaje significativo y autónomo (Tesouro 2005).

Al utilizar el Seminario como estrategia de aprendizaje en alumnos de Biología y Bioquímica, Mariles afirma que durante su desarrollo se produce una acción comunicativa, que permite potenciar a cada miembro del grupo y proveer de una retroalimentación constante durante la experiencia; las disertaciones grupales ayudan a comprender un texto y la comunicación entre los alumnos, de modo que se fomenta el aprendizaje metacognitivo en cada participante (Mariles 2012).

Pozo, al respecto, afirma que la metacognición es fundamental en los procesos de enseñanza-aprendizaje de las ciencias, ya que se ocupa del procesamiento de la información, permitiendo desarrollar habilidades y, además, se interesa por el estudio de las representaciones mentales del sujeto que aprende (Pozo 1999).

En cuanto al proceso de enseñanza y aprendizaje, Martínez enfatiza el rol del querer, del saber y del poder aprender - orientado a la mejoría del rol del docente en la enseñanza estratégica - planteando sugerencias didácticas para su logro, tales como: considerar los conocimientos previos, promover el trabajo en equipo, estimular la autorregulación, explicitar las expectativas de estudiantes y docentes, motivar la participación, utilizar diversos tipos de evaluación, realizar la retroalimentación y la oportunidad de progresar en todas las disciplinas, desde la educación inicial a la universitaria (Martínez 2002).

Con relación a la motivación, Díaz y Hernández reconocen a sus alumnos en su dimensión cognitiva, social y emocional y procuran que ese reconocimiento también esté dirigido al grupo, fomentando así el derecho a ser escuchado y el deber de escuchar en las mismas condiciones. Recomiendan a los maestros incluir en la planificación y desarrollo de contenidos, la ejecución de estrategias cognitivas, metacognitivas y motivacionales, a fin de potenciar en los estudiantes la autonomía, la creatividad y las habilidades de pensamiento que se pueden aplicar a la construcción de conocimientos en los procesos de enseñanza y aprendizaje de Biología Molecular (Díaz y Hernández 1999). 
Osses y Carrasco, en su artículo sobre Módulos Alternativos de Aprendizaje en Biología, afirman que estos: "han contribuido al desarrollo de conocimientos en profundidad, a mejorar la capacidad de reflexionar sobre la forma en que aprenden los estudiantes, a la mejor autorregulación de su propio proceso de aprendizaje y a la práctica de valores a nivel personal y social" (Osses y Carrasco 2013).

\subsection{MEDICIÓN DE LA METACOGNICIÓN}

Mazarella, creó y validó el sistema computarizado Génesis en Venezuela, dirigido al aprendizaje de Biología en estudiantes de educación media, basado en enfoques pedagógicos orientados al desarrollo de la capacidad para aprender, estimular el análisis, la reflexión y la aplicación de los aprendizajes. Se trata de un sistema interactivo multimedia que permite al estudiante avanzar según sus necesidades, controlar su proceso de aprendizaje, contar con retroalimentación y con un registro de interacción de las ejecuciones realizadas bajo la asesoría del profesor. Las variables: aprendizaje de contenido, desarrollo de habilidades metacognitivas y actitud hacia la ciencia, se evaluaron mediante la aplicación de pretest y postest (Mazarella 2006).

Mazarella, utilizando Génesis, realizó una experiencia con estudiantes de educación media diversificada, donde la variable: desarrollo de habilidades metacognitivas, se evaluó mediante aplicación de pretest y postest y los registros de interacción individual que proporciona el sistema. Los resultados evidenciaron que el sistema promueve el desarrollo de habilidades metacognitivas, da la posibilidad de operacionalizar los enfoques pedagógicos vigentes, a través de los recursos que ofrece la informática y da lugar a futuras investigaciones al integrar las Tics en los procesos educativos (Mazarella 2008). Se infiere que, dado que la educación debe preparar debidamente a los ciudadanos del futuro, quienes integrarán una sociedad en cambio continuo, debe promoverse los enfoques pedagógicos para la enseñanza individualizada, la cual, mediante la interacción profesor-estudiantetecnología haga posible la comunicación y la obtención de conocimientos sólidos, útiles y significativos y, simultáneamente, dote a los ciudadanos de aptitudes para aprender durante toda la vida.

Evaluando el efecto de la mediación metacognitiva sobre las estrategias de enseñanza en el área básica de Medicina en Cuba, Alterio y Ruiz, recopilaron información sobre los procesos de pensamiento en la acción docente y las estrategias de enseñanza utilizadas, evaluándose el efecto de la intervención, durante y al final de ella. Inicialmente predominó un estilo de pensamiento poco autorregulado y el uso de estrategias de la enseñanza tradicional; durante y posterior a la mediación, hubo un cambio de actitud consciente al usar estrategias novedosas para enseñar y aprender. Se demostró que los docentes cambiaron su actitud y manera de pensar ante la responsabilidad que conlleva el proceso educativo y la evaluación de su propia práctica pedagógica, perfeccionándose así, el proceso de enseñanza y aprendizaje (Alterio y Ruiz 2010).

Vásquez, incluye en sus aulas de clase a estudiantes discapacitados, los cuales en forma gradual, van adquiriendo destrezas, competencias y habilidades para conocer y controlar conscientemente su aprendizaje (Vásquez 2015). Por otra parte, Ceniceros y Gutiérrez, analizaron las estrategias metacognitivas que utilizan los estudiantes universitarios de la Universidad Pedagógica de Durango, obteniendo resultados de acuerdo con lo planteado por autores, tales como: Vigotsky, Piaget y otros, señalando que las edades de los alumnos (17 
años los estudiantes de pregrado, hasta 52 los estudiantes de postgrado), están directamente relacionadas con el nivel de conciencia que manifestaran en lo que se refiere a sus propios niveles de desarrollo. Aplicaron un cuestionario estructurado en escala Likert que arrojó una confiabilidad de .960 (Alpha de Cronbach), el cual consta de dos partes: la primera tiene datos de los participantes (género, edad y nivel de estudios) y la siguiente consta de dos incisos que corresponden a las dimensiones de la metacognición: autoconocimiento y autorregulación (Ceniceros y Gutiérrez 2009).

En busca de evidencias, las autoras González y Escudero, utilizando un grupo experimental y comparándolo con un grupo control de estudiantes de 15 años, confirmaron que, mediante las actividades de cognición y metacognición, se logra mejorar la autonomía en la disciplina de Química (González y Escudero 2007).

A nivel nacional, Jaramillo y Osses validaron un instrumento diseñado para medir metacognición en términos de conocimiento, experiencias metacognitivas y autorregulación cognitiva, dirigido a estudiantes de $7^{\circ}$ y $8^{\circ}$ Básico de escuelas municipalizadas de alta vulnerabilidad de la Región de la Araucanía, Chile (Jaramillo y Osses 2012).

La revisión bibliográfica descrita, ha mostrado estudios sobre metacognición que, en su mayoría, consideran este constructo en forma global, observándose un escaso número de autores que profundizan en sus dimensiones tales como: Ceniceros y Gutiérrez (Ceniceros y Gutiérrez 2009) y Mariles ( Mariles 2012).

El objetivo de este artículo, en cambio, apunta a presentar los posibles avances experimentados en el desarrollo de la metacognición, por parte de estudiantes chilenos de Segundo Ciclo de Enseñanza Media de Establecimientos Educacionales de Comunas vulnerables de la región de la Araucanía, a partir de una intervención pedagógica que consistió en la aplicación de módulos de aprendizaje en Biología, tomando en cuenta las dimensiones metacognitivas: conocimiento, control y supervisión, planificación, experiencias, evaluación y estrategias. Por tanto, su aporte puede valorizarse desde la perspectiva del análisis de resultados de cada una de dichas dimensiones, en función del aprendizaje de estudiantes que participaron en la investigación.

\section{MÉTODO}

\subsection{DISEÑO}

Desde el punto de vista metodológico, la investigación original fue de carácter mixto (Bericat 1998; Campos 2009); tuvo una duración de tres años $(2010,2011,2012)$ y utilizó un diseño cuantitativo (cuasi experimental) y cualitativo (investigación-acción).

\subsection{INSTRUMENTO}

Respecto del ámbito cuantitativo - a cuyos resultados se refiere este artículo - para el diseño cuasi experimental se utilizó un instrumento de medición de la metacognición. previamente validado.

De acuerdo a la información obtenida en la validación del instrumento (Jaramillo y Osses 2012), la fiabilidad medida mediante consistencia interna (Alpha de Cronbach), correspondió a .860 y la validez de constructo medida mediante análisis factorial 
exploratorio, correspondió a seis factores que explican el 55.2\% de la varianza. Estos resultados, son aceptables para su utilización en el contexto chileno.

Su versión definitiva quedó conformada por 33 ítemes, como se muestra a continuación

\section{Instrumento de Metacognicion}

Nombre:

Curso:

Fecha:

Establecimiento:

Estimado (a) estudiante:

Con el objeto de apoyarte en que mejores tus aprendizajes, a continuación, te presentamos un conjunto de afirmaciones que te solicitamos encarecidamente respondas completo en la forma más honesta posible.

Por favor, marca con una $\mathrm{X}$ la respuesta que más se aproxime a lo que tú piensas o haces.

\begin{tabular}{|c|c|c|c|}
\hline & $\begin{array}{l}\text { Totalmente } \\
\text { de acuerdo }\end{array}$ & $\begin{array}{c}\text { Parcialmente } \\
\text { de acuerdo }\end{array}$ & $\begin{array}{c}\text { Totalmente en } \\
\text { desacuerdo }\end{array}$ \\
\hline \multicolumn{4}{|l|}{$\begin{array}{l}\text { 1. Es bueno descomponer un problema en problemas } \\
\text { más pequeños para resolverlo }\end{array}$} \\
\hline \multicolumn{4}{|l|}{$\begin{array}{l}\text { 2. Yo pienso en diversas maneras para resolver un } \\
\text { problema y luego escojo la mejor }\end{array}$} \\
\hline \multicolumn{4}{|l|}{ 3. Yo imagino el problema para decidir cómo resolverlo } \\
\hline \multicolumn{4}{|l|}{$\begin{array}{l}\text { 4. Yo decido lo que necesito hacer antes de comenzar } \\
\text { una tarea }\end{array}$} \\
\hline \multicolumn{4}{|l|}{ 5. Sé qué pasos debo seguir para resolver un problema } \\
\hline \multicolumn{4}{|l|}{$\begin{array}{l}\text { 6. Voy revisando los objetivos para saber si los estoy } \\
\text { logrando }\end{array}$} \\
\hline \multicolumn{4}{|l|}{$\begin{array}{l}\text { 7. Lo que aprendo me debe servir para comprender } \\
\text { otras cosas }\end{array}$} \\
\hline \multicolumn{4}{|l|}{ 8. Sé que aprendí cuando puedo explicar otros hechos } \\
\hline \multicolumn{4}{|l|}{ 9. Me puedo dar cuenta que no aprendí } \\
\hline \multicolumn{4}{|l|}{$\begin{array}{l}\text { 10. Cuando voy a comenzar una tarea me pregunto qué } \\
\text { quiero lograr }\end{array}$} \\
\hline \multicolumn{4}{|l|}{ 11. Me propongo objetivos con cada tarea } \\
\hline \multicolumn{4}{|l|}{ 12. Me pregunto si lo estoy haciendo bien } \\
\hline \multicolumn{4}{|l|}{$\begin{array}{l}\text { 13. Controlo el tiempo para saber si terminaré todo mi } \\
\text { trabajo en clases }\end{array}$} \\
\hline $\begin{array}{l}\text { 14. Cuando termina la clase me pregunto si pude poner } \\
\text { atención a lo importante }\end{array}$ & & & \\
\hline
\end{tabular}




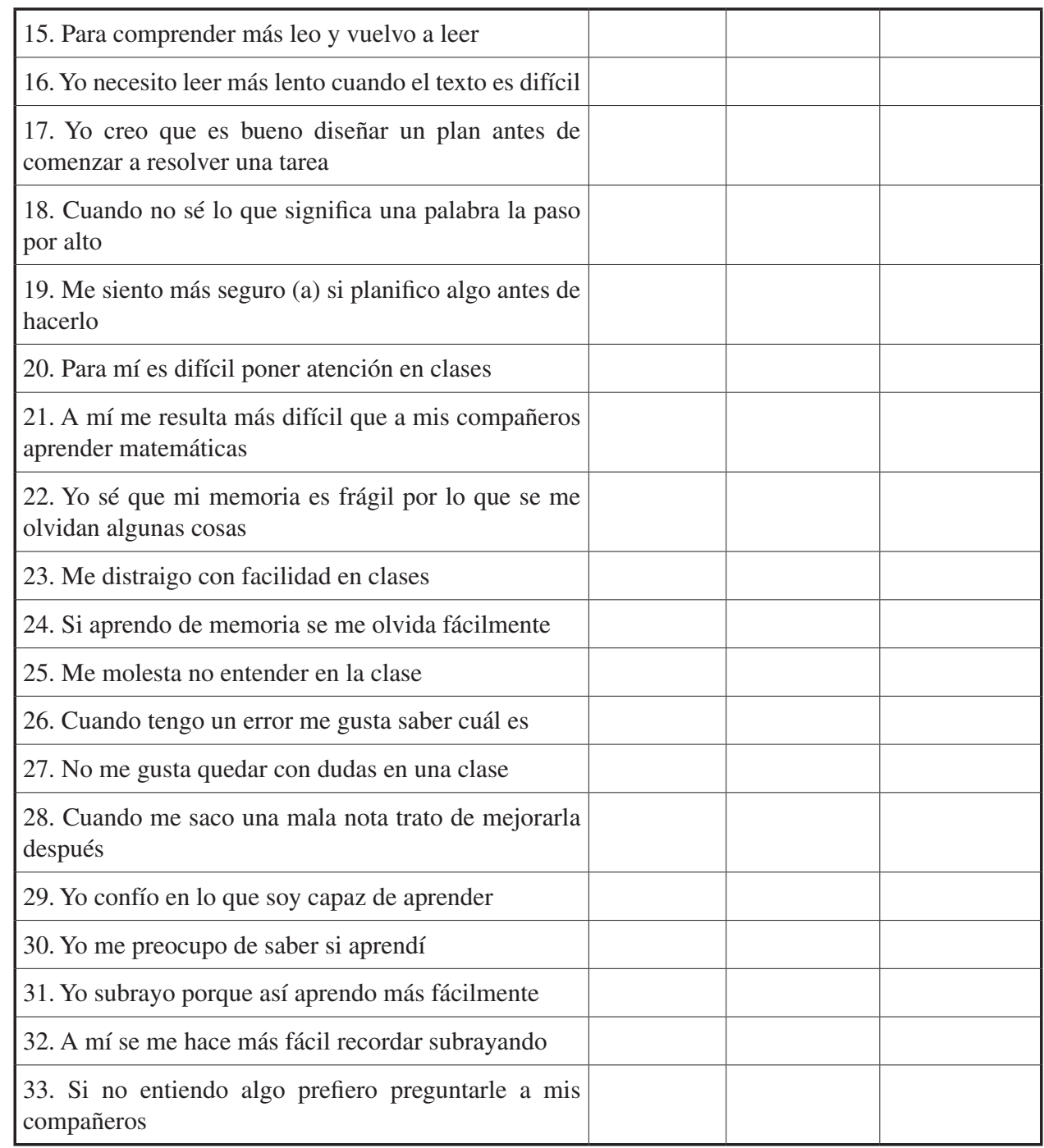

\subsection{INTERVENCIÓN PEDAGÓGICA}

\subsubsection{Estrategia modular}

La investigación consideró la elaboración de Módulos de Aprendizaje por parte de los docentes de los colegios seleccionados, y su aplicación a sus estudiantes de Tercer Año de Enseñanza Media (2010) y a los mismos alumnos, al año siguiente, en Cuarto Año de Enseñanza Media (2011) en la asignatura de Biología, utilizando la Estrategia Modular Alternativa (Osses y Carrasco 2013). La denominación "Alternativa" se refiere a que dichos módulos no están estructurados sobre la base del enfoque conductista a partir del 
cual se originó esta estrategia, sino sobre la base del paradigma humanista sociocognitivo (Román 2010).

El modelo de intervención sociocognitivo empleado, tuvo como propósito general el desarrollo de capacidades y valores implícitos en un aprendizaje de calidad - caracterizado este por ser profundo, integral y autónomo (Osses 2013) - mediante la práctica de estrategias de aprendizaje adecuadas por parte de los estudiantes de Segundo Ciclo de Enseñanza Media y de adecuadas estrategias de enseñanza por parte de profesores de Biología, con especial énfasis en el desarrollo de la metacognición de los estudiantes.

Los Módulos de Aprendizaje, material básico para lograr el aprendizaje de los alumnos de Segundo Ciclo de Enseñanza Media, fueron elaborados por los profesores de las Comunas participantes; para ello, contaron con un período de capacitación en Biología y en Didáctica (I Semestre 2010 y Primer Semestre 2011), a fin de desempeñar en forma adecuada su rol de mediadores del proceso de aprendizaje. Apoyados por el equipo de investigación, incorporaron a los Módulos, actividades orientadas a desarrollar capacidades desglosadas a nivel de destrezas, conjuntamente con las actividades orientadas a desarrollar valores, desglosados a nivel de actitudes. A partir de la interacción estudiante-módulo y las implicaciones educativas del entorno sociocultural, emergieron los datos del proyecto, que corresponden, principalmente, a los alumnos, centro del proceso educativo, y también a los profesores, en su rol de mediadores del aprendizaje.

\subsubsection{Estructura de los módulos alternativos de aprendizaje}

Cada Módulo Alternativo estuvo conformado por Guías de Aprendizaje que incluyeron: a) Objetivos: planteados en términos de capacidades y valores; b) Contenidos: formulados como medios para lograr los objetivos, referidos a temáticas que, por su gran carga valórica, se prestan para el tratamiento conjunto de los ámbitos cognitivo y afectivo; c) Estrategias didácticas: medios para lograr los objetivos en términos de actividades; entre otras, incluyeron la realidad concreta del alumno, su entorno socio-cultural, su vida familiar, sus aspiraciones y valores personales y de su grupo de pertenencia, su explicitación de ideas en forma individual, grupal o conjunta, actividades del alumno solo, con carácter de reflexión personal, discusiones, trabajos prácticos o visitas a distintas instancias extra-aula en grupos pequeños, salidas a terreno del grupo curso y profesor para estar en contacto directo con el problema en estudio y conversaciones con las familias sobre la temática abordada. El docente, mediador del aprendizaje, promovió la construcción o reconstrucción de conocimientos por parte de los estudiantes. A fin de que la metacognición y el trabajo grupal se incorporaran realmente al proceso educativo, se explicitó su presencia en cada una de las Guías de Aprendizaje que conformó el Módulo; d) Evaluación: los módulos se iniciaron con una indagación sobre los conocimientos previos de los estudiantes en calidad de evaluación diagnóstica; se enfatizó la evaluación formativa en los ámbitos cognitivo y afectivo durante el desarrollo de las clases, finalizando cada módulo con una evaluación de carácter metacognitivo. Se realizó evaluación sumativa utilizando técnicas o instrumentos cualitativos y cuantitativos coherentes con el enfoque sociocognitivo del aprendizaje que orientó la investigación, entre otros: listas de control, coevaluación, autoevaluación, debates, disertaciones y entrevistas; todos los instrumentos fueron elaborados y aplicados sobre la base de una pauta orientadora.

A manera de ejemplo, a continuación, se presenta una guía de un módulo de aprendizaje alternativo para tercer año de Enseñanza Media. 


\section{MÓDULO DE APRENDIZAJE ALTERNATIVO PARA TERCER AÑO DE EDUCACIÓN MEDIA. BIOLOGÍA E HIGIENE NERVIOSA}

\section{TEMA: DROGAS Y TOXICOMANÍA}

\section{OBJETIVOS:}

- Conocer y comprender qué son las drogas, cuáles son y sus efectos en el organismo.

- Relacionar nueva información con conocimientos previos.

- Fortalecer vínculos de apoyo e interrelación entre pares.

- Tomar conciencia de sus propios procesos cognitivos.

- Desarrollar la capacidad de autorregular su aprendizaje.

- Aplicar los conocimientos adquiridos a distintos contextos.

- Desarrollar valores tales como: la responsabilidad, el respeto y la participación.

\section{PRESENTACIÓN}

Estimado(a) estudiante:

Con mucho afecto ponemos en tus manos estos Módulos de Aprendizaje en Biología, para que, a través de ellos, tengas la oportunidad de conocer un tema vivencial, como es Biología e Higiene Nerviosa y sus implicancias, como asimismo, reflexionar y dialogar sobre ellas con tus compañeros (as) y personas de la comunidad en que estás inserto, especialmente, con tu familia.

A través del módulo pretendemos que construyas conocimientos, puedas compartir sentimientos y creencias con respecto al alcohol y a otras drogas y dialogar sobre el tema, teniendo la oportunidad de aprender a tomar decisiones responsables.

Durante el proceso de aprendizaje en Biología, tú serás el protagonista, es decir, lo que aprendas dependerá de ti, de tu entusiasmo, responsabilidad y creatividad. Tu profesor(a) será un guía, de modo que podrás, con tranquilidad, acoger las actividades sugeridas y llevarlas a la práctica, proponer actividades diferentes y, en general, elegir entre alternativas en variados temas.

Es preciso señalar que, los módulos no se agotan en el mero conocimiento científico. En efecto, a lo largo de su desarrollo, se da gran importancia al aspecto de formación, especialmente en responsabilidad personal y social, lo cual se refleja, claramente, en las Guías de Aprendizaje.

Esperamos que el trabajo con este material, signifique para ti una oportunidad de profundizar en temas de enorme trascendencia social y, a través del desarrollo de los ámbitos cognitivo y valórico, contribuya a tu formación integral como persona comprometida con la armonía con tu medio natural y sociocultural.

\section{¿QUÉ SABES SOBRE LAS DROGAS?}

Te invitamos a recordar y reflexionar sobre los conocimientos adquiridos en tu familia y entorno. Te proponemos responder las preguntas que se plantean a continuación, poniendo énfasis en el fundamento de cada una de ellas. Ten presente que tus conocimientos previos permitirán construir conocimientos nuevos sobre una base más sólida, forjar un estilo de 
vida y adaptarte sanamente a cada una de las áreas de la vida cotidiana.

Responde las siguientes afirmaciones, colocando frente a cada afirmación Verdadero (V) o Falso (F) y justifica las respuestas falsas donde corresponda.

Preguntas V F Justifica las Falsas.

1.- El consumo de drogas es sólo un problema de los jóvenes.

2.- Las personas consumen drogas sólo cuando tienen problemas.

3.- Los no fumadores no sufren los efectos del tabaco.

4.-Todas las drogas producen los mismos efectos.

5.- Es importante que los niños prueben bebidas alcohólicas en compañía de sus familiares.

6.- En relación a las drogas, existen los que consumen y los que son dependientes.

7.- Si una droga es natural, es decir, no procesada, es menos dañina que una sintética.

8.- Si no se bebe alcohol en una fiesta es, generalmente, una fiesta "fome"

9.- Si vendo o regalo drogas a mis amigos o conocidos, no cometo ningún delito.

10.- Estar informado acerca de las drogas, ayuda a optar por un estilo de vida saludable.

11.- El alcohol y el tabaco producen enfermedades.

\section{GUÍA DE APRENDIZAJE ¡APRENDAMOS SOBRE LAS DROGAS!}

\section{ACTIVIDAD N 1: "LEER COMPRENSIVAMENTE"}

\section{MATERIALES}

Historia de vida, Anexos $\mathrm{N}^{\circ}$ 1, 2, 3, 4. Texto del Alumno. Aprendamos a Aprender. Biología Humana. Higiene Nerviosa. Módulo para el estudiante $3^{\circ}$. 2011. Osses, S.; González, M; Manríquez, M., y otros recursos incluyendo los computacionales.

a) Se agrupan de a cuatro estudiantes y leen en silencio el caso dado.

b) Subrayan las principales ideas y hacen inferencias sobre el contenido del material utilizado.

c) Comentan y discuten las posibles causas que conducen al consumo de drogas.

\section{ACTIVIDAD N²: "REFLEXIONAR"}

a) ¿Cuáles decisiones, que tomó o dejó de tomar el protagonista de la historia de vida en el pasado, podrían haberle evitado llegar al problema?

b) ¿Qué decisión le ayudaría a que le fuera bien en su vida?

c) ¿Qué factores influyen que, en este caso, haya riesgo de abuso de alcohol y drogas?

d) Plenario: cada grupo presenta sus conclusiones, se hace una pausa para detenerse en los desacuerdos, buscando los conflictos implícitos y, posteriormente, las soluciones.

e) Cierre: Al finalizar la actividad, se analiza y se hace una síntesis de los factores de riesgo que pueden llevar al consumo de drogas. 


\section{ACTIVIDAD N 3: “COMPARAR, FORMULAR HIPÓTESIS, INTERPRETAR Y PLANTEAR CONCLUSIONES",}

a) Forman grupos de cuatro estudiantes e investigan en internet acerca de los estudios de CONACE sobre el consumo de drogas en escolares chilenos.

b) Seleccionan un estudio reciente y otro de años anteriores, con el fin de comparar tablas de datos y gráficos y formulan hipótesis que expliquen la distribución y evolución del consumo de drogas.

c) Interpretan y comparan datos, emiten juicios críticos respecto a la magnitud del fenómeno.

d) Determinan cuáles son los factores de riesgo más importantes que inciden en el consumo de drogas en la población escolar.

e) Concluyen sobre la base de los datos analizados

f) Hacen puesta en común y socializan sus conclusiones.

\section{ACTIVIDAD N 4: “INVESTIGAR, COMUNICAR, DEBATIR Y VALORAR”}

El Debate consiste en un intercambio informal de ideas e información sobre un tema que se presta a la controversia, realizado por un grupo, bajo la conducción dinámica y estimulante de un coordinador o moderador.

En grupos de 6 estudiantes investigan temas contrapuestos, entregados por el profesor, que están relacionados con el efecto de las drogas lícitas e ilícitas en el cuerpo humano.

Cada grupo investiga sobre la postura que le corresponderá defender y es responsable de:

a) Estudiar lo que se le propone y ampliar la información sobre el tema.

b) Separar la información verificable de las opiniones personales.

c) Disentir de lo que considere erróneo o falso.

d) Seleccionar y diferenciar lo relevante de lo secundario.

e) Sacar conclusiones propias. Adicionalmente, se tiene que preparar los materiales de apoyo (cuadros estadísticos, mapas conceptuales, grabaciones, etc.)

f) Deben llegar a la sala de clases, bien preparados. Es importante recurrir a argumentos lógicos, para defender un punto de vista o una idea. El tipo de lenguaje debe ser argumentativo.

g) Cada grupo deberá elegir un representante que comenzará el debate. Luego, todos los participantes deben debatir según el turno que le dé el moderador.

h) El debate durará 20 minutos. Cada expositor dispondrá de tres minutos para cada una de sus intervenciones. La intervención puede ser interrumpida, siempre y cuando el expositor lo permita y se le solicite respetuosamente.

i) Terminado el debate, el Secretario tratará de que la asamblea llegue a las conclusiones.

j) Como actividad de cierre, realizan difusión del tema a través de un tríptico preparado por ellos a otros cursos, colegio, etc. 


\section{ACTIVIDAD DE TÉRMINO DEL MÓDULO REFLEXIONA SOBRE TU APRENDIZAJE}

Nombre:

Fecha: ....

Establecimiento:

Curso:

¿Qué aprendí? ¿Por qué creo que lo aprendí?

¿Cómo lo aprendí? ¿Tuve otras alternativas para aprender? ¿por qué seguí ese camino?

\subsection{MUESTRA}

El proceso de selección de los estudiantes se realizó mediante un muestreo no probabilístico. Los alumnos que participaron en el estudio respondieron el cuestionario en dos ocasiones: al comienzo de la intervención educativa, con 106 estudiantes y dos años más tarde, una vez finalizada la intervención, con 101 alumnos. Participaron estudiantes de ambos sexos (48.5\% hombres, $51.5 \%$ mujeres) y edades comprendidas entre los 16 y 18 años. Los estudiantes pertenecían a Segundo Ciclo de Enseñanza Media de Liceos Municipalizados de cuatro comunas de la región de la Araucanía de Chile. La distribución según comunas fue la siguiente:

Tabla 1. Distribución de la muestra según Comuna

\begin{tabular}{|l|c|c|c|c|}
\hline & \multicolumn{2}{|c|}{ Pretest } & \multicolumn{2}{c|}{ Postest } \\
\hline & Frecuencia & Porcentaje válido & Frecuencia & Porcentaje válido \\
\hline Lonquimay & 17 & 16.0 & 24 & 23.8 \\
\hline Carahue & 35 & 33.0 & 30 & 29.7 \\
\hline Saavedra & 25 & 23.6 & 23 & 22.8 \\
\hline Collipulli & 29 & 27.4 & 24 & 23.8 \\
\hline Total & 106 & $100 \%$ & 101 & $100 \%$ \\
\hline
\end{tabular}




\subsection{ANÁLISIS DE DATOS}

Se llevó a cabo mediante el software SPSS 20.0. Para medir las diferencias generadas por la intervención, se aplicó la prueba t de Student (Hernández, Fernández y Baptista 2010) con el objeto de realizar un análisis preliminar de los promedios obtenidos por los estudiantes, según el test inicial (pretest) al comenzar la intervención, y el test final (postest), al terminar la intervención. Una vez comprobadas las diferencias de medias, se utilizó el estadístico d; esta medida se entiende como la razón entre la diferencia de medias entre medición postest y pretest, y la desviación estándar. Una orientación para interpretar este estadístico puede encontrarse en Morales donde un valor de 0.20 es considerada una diferencia pequeña, 0.50 como moderada y 0.80 como grande (Morales 2008). El uso del estadístico d permite realizar comparaciones entre dos mediciones en distintos periodos de tiempo. Esto se realizó totalizando ambas puntuaciones de metacognición de los estudiantes.

\section{RESULTADOS}

\subsection{ANÁLISIS DESCRIPTIVO}

El análisis descriptivo de los ítemes del cuestionario sobre metacognición, se realizó calculando los puntajes promedio y la desviación estándar de cada una de las sentencias en las dos mediciones: pretest y postest. Como se observa en la Tabla 2, la mayoría de los ítemes medidos durante el pretest presenta valores promedio $<=2$. Si se observa la columna postest, se encuentran valores mayores o iguales a dos puntos, evidenciando un aumento significativo respecto de la segunda medición. Resulta interesante el comportamiento de los ítemes 13, 18 y 20 que presentan una media mayor en el pretest.

Este análisis descriptivo de la escala permite pronosticar que se encontrarán diferencias significativas en el análisis comparativo.

Tabla 2. Medias y desviaciones estándar según mediciones pretest y postest

\begin{tabular}{|l|c|c|c|c|}
\hline & \multicolumn{2}{|c|}{ Pretest } & \multicolumn{2}{c|}{ Postest } \\
\hline & Media & Desv. Estándar & Media & Desv. Estándar \\
\hline It.1 & 1.80 & .668 & 2.67 & .513 \\
\hline It.2 & 1.35 & .554 & 2.61 & .586 \\
\hline It.3 & 1.56 & .619 & 2.50 & .626 \\
\hline It.4 & 1.51 & .605 & 2.59 & .533 \\
\hline It.5 & 1.75 & .549 & 2.40 & .531 \\
\hline It.6 & 1.70 & .692 & 2.36 & .672 \\
\hline It.7 & 1.15 & .385 & 2.83 & .471 \\
\hline
\end{tabular}




\begin{tabular}{|l|c|c|c|c|}
\hline It.8 & 1.40 & .549 & 2.67 & .550 \\
\hline It.9 & 1.36 & .592 & 2.70 & .541 \\
\hline It.10 & 1.94 & .618 & 2.15 & .592 \\
\hline It.11 & 1.93 & .624 & 2.24 & .666 \\
\hline It.12 & 1.61 & .658 & 2.50 & .594 \\
\hline It.13 & 2.10 & $.613 c$ & 2.06 & .577 \\
\hline It.14 & 1.83 & .673 & 2.19 & .529 \\
\hline It.15 & 1.37 & .559 & 2.73 & .535 \\
\hline It.16 & 1.46 & .652 & 2.66 & .613 \\
\hline It.17 & 1.67 & .599 & 2.43 & .558 \\
\hline It.18 & 2.21 & .675 & 1.68 & .688 \\
\hline It.19 & 1.46 & .572 & 2.46 & .597 \\
\hline It.20 & 2.11 & .681 & 1.99 & .718 \\
\hline It.21 & 1.92 & .817 & 2.14 & .763 \\
\hline It.22 & 1.78 & .665 & 2.03 & .656 \\
\hline It.23 & 1.70 & .678 & 2.05 & .702 \\
\hline It.24 & 2.08 & .706 & 2.22 & .716 \\
\hline It.25 & 1.54 & .638 & 2.50 & .689 \\
\hline It.26 & 1.24 & .511 & 2.65 & .591 \\
\hline It.27 & 1.53 & .609 & 2.51 & .610 \\
\hline It.28 & 1.34 & .572 & 2.69 & .596 \\
\hline It.29 & 1.27 & .529 & 2.63 & .561 \\
\hline It.30 & 1.55 & .574 & 2.40 & .665 \\
\hline It.31 & 1.76 & .692 & 2.41 & .686 \\
\hline It.32 & 1.86 & .718 & 2.46 & \\
\hline It.33 & 1.46 & .655 & & \\
\hline & & & .50 & \\
\hline
\end{tabular}

\subsection{EVIDENCIAS COMPARATIVAS}

Para evaluar si la intervención pedagógica generó un avance en los niveles de metacognición, se realizó un análisis comparativo entre los puntajes obtenidos por los estudiantes en el pretest y el postest. Para hacer factible dicha comparación, considerando que las escalas tenían puntuaciones diferentes, se optó por estandarizar las variables. Una vez finalizado este proceso, se obtuvo las puntuaciones separadas para estimar sus diferencias. Además, se optó por realizar una prueba t de Student para establecer si las 
diferencias eran estadísticamente significativas. Como puede apreciarse en el Gráfico 1, prácticamente la totalidad de las dimensiones de la metacognición presentaron diferencias significativas, que fueron ratificadas por la prueba $\mathrm{T}$ de Student al confirmar valores $\mathrm{p}<.001$ en las dimensiones; conocimiento $(\mathrm{t}=-24.402 ; \mathrm{p}<.001)$, control y supervisión $(\mathrm{t}=-6.198 ; \mathrm{p}<.001)$, planificación $(\mathrm{t}=-20.448 ; \mathrm{p}<.001)$, evaluación $(\mathrm{t}=-20.288 ; \mathrm{p}<.001)$ y estrategias $(\mathrm{t}=-10.485 ; \mathrm{p}<.001)$. La única dimensión que se mantuvo sin diferencias tras la intervención educativa fue la dimensión Experiencias $(\mathrm{t}=-1.864 ; \mathrm{p}=.064)$, la cual no fue incluida en el siguiente nivel de análisis.

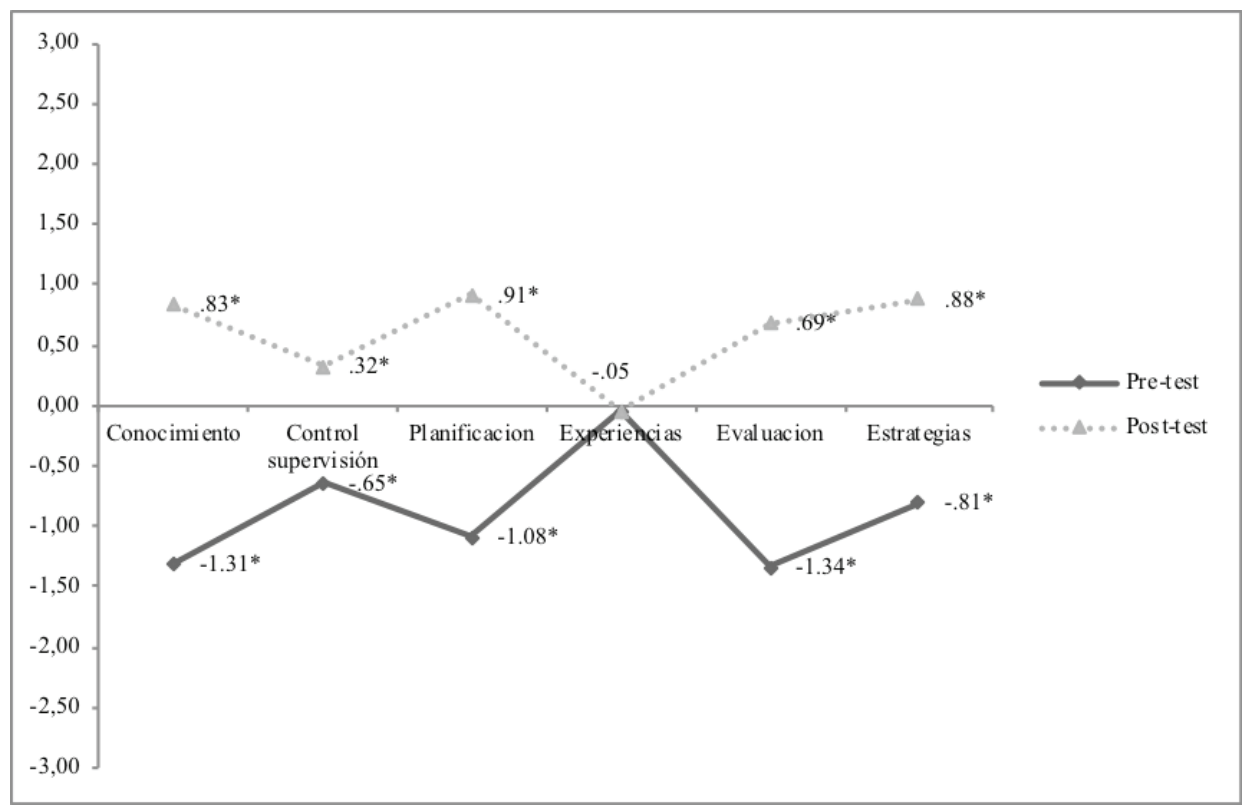

Gráfico 1. Análisis comparativo dimensiones metacognición según pretest, postest

Las variables fueron estandarizadas. Valores con asterisco simbolizan $p<.001$, tras aplicación de la prueba de diferencia de medias, T de Student.

\subsection{EVIDENCIAS DE MAGNITUD}

Una vez calculadas las diferencias obtenidas en las distintas dimensiones de la metacognición, se realizó un análisis para evaluar la magnitud de dichas diferencias y establecer cuáles eran las dimensiones con mayor cambio tras la intervención educativa. Como puede observarse en la Tabla 3, la subescala Conocimiento, presenta la mayor distancia entre la medición pretest y postest $(\mathrm{d}=2.45)$, en segundo lugar, le sigue la dimensión Evaluación $(\mathrm{d}=2.03)$, luego Planificación $(\mathrm{d}=1.63)$, Estrategias $(\mathrm{d}=1.04) \mathrm{y}$, finalmente, la escala de Control-Supervisión $(\mathrm{d}=.62)$. Todas las dimensiones señaladas obtuvieron una magnitud de diferencia grande (Morales 2008). 
Estudios Pedagógicos XLIV, N $^{\circ}$ 1: 69-88, 2018

EFECTO DE UNA ESTRATEGIA PEDAGÓGICA SOBRE EL DESARROLLO METACOGNITIVO DE ADOLESCENTES CHILENOS

Tabla 3. Medición del tamaño del efecto según dimensiones de metacognición

\begin{tabular}{|l|c|c|c|c|c|}
\hline & Conocimiento & Control supervisión & Planificación & Evaluación & Estrategias \\
\hline Media postest & 23.34 & 11.19 & 11.93 & 15.39 & 7.36 \\
\hline $\begin{array}{l}\text { Desviación estándar } \\
\text { postest }\end{array}$ & 3.16 & 2.05 & 1.74 & 2.73 & 1.65 \\
\hline Media pretest & 13.54 & 9.42 & 8.18 & 8.49 & 5.09 \\
\hline $\begin{array}{l}\text { Desviación estándar } \\
\text { pretest }\end{array}$ & 2.45 & 1.97 & 1.50 & 2.04 & 1.42 \\
\hline Tamaño efecto & 2.45 & .62 & 1.63 & 2.03 & 1.04 \\
\hline
\end{tabular}

\section{DISCUSIÓN E INTERPRETACIÓN DE LOS RESULTADOS}

Respecto de la evaluación de la metacognición, tema central de este artículo, Saldaña y Aguilera afirman que, de acuerdo al desarrollo alcanzado por el conocimiento de este constructo, ninguna técnica sola puede proporcionar una valoración precisa de los procesos metacognitivos: conocimiento del conocimiento y regulación cognitiva (Saldaña y Aguilera 2003). De esta afirmación se infiere que, es recomendable combinar técnicas e instrumentos de evaluación para medirlo. Así, por ejemplo, podría utilizarse la ejecución de alguna tarea por parte del estudiante, simultáneamente con un test que mida su percepción sobre la misma y una entrevista que permita profundizar sobre ella. En esta forma, el cruce de indicadores de la metacognición permitirá al investigador obtener una aproximación, probablemente más válida, acerca de dicho constructo.

Es así como, Sandi-Urena y Cooper, en una investigación sobre Enseñanza de la Química, utilizó un diseño secuencial de métodos mixtos, combinando un componente cuantitativo y un componente cualitativo. El primero, correspondió a la estrategia multi-método conformada por el Inventario de Actividades Metacognitivas (MCAi) y el instrumento automatizado, Interactive MultiMedia Exercises (IMMEX), capaz de recoger información sobre estrategias, de manera simultánea a la ejecución de la tarea; en conjunto, estos métodos dieron cuenta de lo que los participantes informan hacer cuando resuelven problemas y lo que en realidad hacen cuando los resuelven; en cuanto al componente cualitativo, se refirió a un estudio fenomenológico que, en coherencia con dicha metodología, permitió obtener información desde los marcos de referencia de los participantes (Sandi-Urena y Cooper 2010).

El componente cuantitativo detectó los cambios generados por la intervención, en este caso, la participación de los estudiantes en el laboratorio cooperativo, y midió las variaciones en los parámetros correspondientes a la estrategia multi-método: puntaje MCAi, estrategia metacognitiva y habilidad para resolver problemas, lográndose establecer que, de acuerdo con sus estrategias en las soluciones de problemas de química en línea (calidad metacognitiva de la estrategia en IMMEX), los estudiantes en el laboratorio mostraron mayor uso de habilidades metacognitivas. Asimismo, este grupo demostró ser capaz de resolver mayor cantidad de problemas de alto nivel de dificultad, en comparación con los estudiantes que no participaron de la intervención. 
Del mismo modo, en el caso de la investigación que originó este artículo (Osses 2013), además del instrumento cuantitativo utilizado antes y después de la intervención pedagógica - cuya diferencia de magnitud midió el cambio producido en el desarrollo de la metacognición - se utilizó técnicas de recolección de datos cualitativas, tales como: observación participante y grupo focal, orientadas a triangular la información a objeto de validarla, lo que será objeto de futuras publicaciones.

En este caso, la mayor diferencia producida entre pretest y postest correspondió a Conocimiento, seguido de Evaluación, Planificación, Estrategias y Control y Supervisión, teniendo todas estas dimensiones, una magnitud de diferencia grande según la escala de Morales (Morales 2008).

Los resultados obtenidos estarían indicando que, en los estudiantes, el conocimiento metacognitivo y su autorregulación, fueron más profundos una vez finalizada la intervención pedagógica, que a su inicio; en otras palabras, se ha producido un avance de la metacognición en los alumnos durante el proceso educativo, que se puede atribuir, al menos en parte, al constante proceso reflexivo generado por la intervención pedagógica, antes, durante y después de ejecutar la tarea.

La dimensión, Experiencias Metacognitivas - la cual según Efklides ha recibido muy poca atención respecto de sus implicancias en el proceso de aprendizaje - fue la única que no experimentó diferencias significativas en su avance (Efklides 2006).

El análisis de esta dimensión, entendida como: "Sentimientos, estimaciones o juicios relativos a las tareas de aprendizaje, sobre cómo tiene lugar el procesamiento cognitivo, así como su resultado" (Efklides 2009: 76), permite darse cuenta que en ella está presente, en gran medida, el componente afectivo. En efecto, los sentimientos y juicios acerca del conocimiento, que ocurren durante el proceso de aprendizaje, se refieren, en los primeros, a: dificultad, confianza, satisfacción y familiaridad y, en los segundos, a búsqueda de solución correcta, a demandas cognitivas incluyendo valoración del tiempo y a esfuerzo requerido.

Crescenzi (Crescenzi 2016) ejemplifica el carácter afectivo de las Experiencias Metacognitivas refiriéndose al tiempo que un estudiante empleó en la tarea de buscar artículos y la respuesta afectiva (presión de la hora) que le acompañó: "después del primer tópico, sentí la presión inmediatamente. Se agudizó cuando pude sentir que la hora pasaba y yo sólo tenía 5 o 6 artículos" (Crescenzi 2016). "Sentí la mayor presión alrededor de la mitad del trabajo sobre el segundo artículo, del cual yo no había encontrado mayor información aún y tenía el sentimiento que se estaba agotando el tiempo. Así, empecé a leer rápidamente para terminar la tarea" (Crescenzi 2016).

Otro estudiante sin límite de tiempo para realizar su tarea, expresó sentirse nervioso respecto del tiempo durante la sesión experimental: "Sentí la presión del tiempo durante la primera tarea cuando tenía que encontrar algunos artículos. Estuve nervioso durante todo el proceso, me habría gustado tomarme más tiempo que el esperado 1,5 horas" (Crescenzi 2016).

Teniendo en cuenta las aseveraciones anteriores y considerando, además, la estrecha relación existente entre activación de los procesos cognitivos, estrategias utilizadas en el proceso de enseñanza y aprendizaje y motivación de los estudiantes, se podría inferir que, el menor desarrollo de la dimensión Experiencias Metacognitivas - la cual acompaña a los alumnos en sus procesos metacognitivos - podría deberse, entre otras razones, a que las estrategias utilizadas para motivar su aprendizaje en esta dimensión, fueron insuficientes 
o inadecuadas, por lo cual, no activaron los procesos cognitivos de los estudiantes en una magnitud tal, que les permitiera lograr plenamente el aprendizaje esperado.

\section{CONCLUSIONES}

A partir de los resultados obtenidos en el ámbito de investigación correspondiente a este artículo, se puede afirmar que:

Se ha realizado una investigación de tres años de duración en Enseñanza de las Ciencias, con alumnos de Segundo Ciclo de Educación Media de 4 comunas vulnerables de la Región de la Araucanía, Chile, orientada a desarrollar en los estudiantes, las capacidades y valores involucrados en un aprendizaje de calidad - es decir, profundo, integral y autónomo mediados por el profesor de Biología, con énfasis en el desarrollo de procesos metacognitivos.

En dicha investigación, se realizó una intervención pedagógica que consistió en la aplicación de módulos de aprendizaje en Biología que incorporaron la dimensión metacognitiva en cada una de las guías de aprendizaje puestas en práctica por los estudiantes.

De carácter mixto, utilizó diseños metodológicos y técnicas de recolección de datos cualitativos y cuantitativos.

En este artículo - que presenta los resultados del ámbito cuantitativo - se analizaron los posibles avances en el desarrollo de la metacognición en la muestra de estudiantes que participó en la investigación.

En el ámbito cuantitativo, dado que se trataba de cursos previamente conformados, se utilizó un diseño cuasi experimental, para lo cual, se aplicó un pretest y un postest, antes y después de la intervención pedagógica, respectivamente.

A partir del análisis comparativo realizado entre los puntajes obtenidos en el pretest y el postest por los estudiantes, se concluye que todas las dimensiones metacognitivas, excepto Experiencias, mostraron cambios estadísticamente significativos.

La evaluación de la magnitud de las diferencias producidas entre pretest y postest, mostró que la mayor diferencia entre ambos momentos del proceso, correspondió a Conocimiento, seguido de Evaluación, Planificación, Estrategias y Control-Supervisión, teniendo todas las dimensiones, una magnitud de diferencia grande según la escala de Morales (Morales 2008).

En cuanto a la dimensión Experiencias Metacognitivas - cuya diferencia entre postest - pretest no fue significativa - de acuerdo a la discusión realizada, debería revisarse en términos de las estrategias de enseñanza y aprendizaje utilizadas para lograr su dominio y, si los resultados lo sugirieran, proceder a modificarlas, a fin de hacerlas más pertinentes a la realidad de los estudiantes y, con ello, activar en mayor medida sus procesos cognitivos, y así, obtener éxito en su proceso de aprendizaje.

Considerando que, en la literatura científica disponible, se encontró escaso número de investigaciones referidas a las dimensiones que conforman la metacognición, el aporte del presente artículo se refiere, principalmente, a la medición de cada una de las dimensiones de este constructo, cuyos resultados han mostrado en forma específica, en qué ámbitos metacognitivos los estudiantes presentaron mayores fortalezas y cuáles sería conveniente reforzar en futuras intervenciones pedagógicas.

Por último, a partir de los resultados obtenidos y, teniendo en cuenta las limitaciones del diseño cuasi experimental, se puede afirmar que la metodología adoptada, en general, 
ha impactado positivamente en el proceso educativo de los estudiantes, especialmente, en aquellos aspectos que van más allá del aprendizaje mismo, es decir, en la reflexión sobre los propios procesos cognitivos y su autorregulación, o en otras palabras que, a través de la intervención pedagógica realizada, se ha logrado desarrollar activos procesos metacognitivos en los estudiantes que participaron en la investigación.

\section{REFERENCIAS BIBLIOGRÁFICAS}

Alterio, G. y Ruiz, C. (2010). Mediación metacognitiva, estrategias de enseñanza y procesos de pensamiento del docente de Medicina. Educación Médica Superior, vol. 24 (1), 25-32.

Bericat, E. (1998). La investigación de los métodos cuantitativo y cualitativo en la investigación social. Significado y medida. Barcelona: Ariel.

Brown, A. L. y Smiley, S. (1978). The development of strategies for studying texts. Child Development. Hillsdale NJ: Erlbaum.

Campos, A. (2009). Métodos mixtos de investigación. Integración de la investigación cuantitativa y la investigación cualitativa. Bogotá: Magisterio.

Ceniceros, D. y Gutiérrez, D. (2009). Las habilidades metacognitivas en los estudiantes de la Universidad Pedagógica de Durango. Psicogente, vol. 12 (21), 29-37.

Crescenzi, A. (Julio de 2016). Metacognitive Knowledge and Metacognitive Regulation in TimeConstrained Information Search. En R. Perego y F. Sebastiani (Presidencia). ACM SIGIR Conference, Pisa, Italy.

De Bullaude, M., Córdoba, L., Torres, M. y De Morán, J. (2008). Análisis de Metodologías de Estudio en Química Inorgánica. Formación Universitaria, vol. 1 (6), 29-34.

Díaz, F. y Hernández, G. (1999). Estrategias docentes para un aprendizaje significativo. Una interpretación constructivista. México, D. F.: McGraw Hill.

Efklides, A. (2006) Metacognition and affect: What can metacognitive experiences tell us about the learning process? Educational Research Review, vol. 1 (1), 3-14.

Efklides, A. (2009). The role of metacognitive experiences in the learning process. Psicothema, vol. $21(1), 76-82$.

Flavell, J. (1976). Metacognitive aspects of problem solving. En L. B. Resnik. (Ed.), The nature of intelligence (pp. 231-235). Hillsdale, N.J., USA: Erlbaum.

González, S. y Escudero, C. (2007). En busca de la autonomía a través de las actividades de cognición y metacognición en Ciencia. Revista Electrónica de Enseñanza de las Ciencias, vol. 6 (2), 310330.

Hernández, R., Fernández, C. y Baptista, P. (2010). Metodología de la Investigación. México, D. F.: McGraw Hill.

Jaramillo, S. y Osses, S. (2012). Validación de un Instrumento sobre Metacognición para Estudiantes de Segundo Ciclo de Educación General Básica. Estudios Pedagógicos, vol. 38 (2), 117-131.

Jerónimo, L. y Ayala, J. (2011). Enseñanza de las ciencias naturales, la importancia de la relación pedagógica en la clase de biología molecular. Orinoquia, vol. 15 (2), 215-222.

Mariles, S. (2012). El Seminario como fuente de aprendizaje cooperativo en el desarrollo de las Ciencias Biológicas. Horizontes Pedagógicos, vol. 14 (1), 141-155.

Martínez, R. (2002). Aprender: Necesaria Unión entre el Querer, el Saber y el Poder. Revista de Pedagogía, vol. 23 (68), 477-494.

Mateos, M. (2002). Metacognición y educación. Buenos Aires: Aique.

Mazarella, C. (2006). Desarrollo y validación de un sistema computarizado para el aprendizaje de un contenido de Genética. Investigación y Postgrado, vol. 21 (2), 11-42.

Mazarella, C. (2008). Desarrollo de habilidades metacognitivas con el uso de las Tics. Investigación 
y Postgrado, vol. 23 (2), 175-204.

Morales, P. (2008). Estadística aplicada a las Ciencias Sociales. Madrid: Universidad Pontificia Comillas.

Osses, S. (2013). Hacia un aprendizaje de calidad en la educación científica. Estrategias didácticas para el desarrollo de capacidades y valores (Informe Final Proyecto Fondecyt 1100378). Universidad de La Frontera, Temuco, Chile.

Osses, S. y Jaramillo, S. (2008). Metacognición: Un camino para aprender a aprender. Estudios Pedagógicos, vol. 34 (1), 187-197.

Osses, S. y Carrasco, L. (2013). Módulos Alternativos en la Enseñanza de las Ciencias. Estrategia didáctica orientada al logro de aprendizajes significativos. Formación Universitaria, vol. 6 (3), $39-52$.

Paz, H. (2011). ¿Cómo desarrollar la metacognición en la educación superior mediante la resolución de problemas? Ingeniería e Investigación, vol. 31 (1), 213-223.

Pérez de Albérniz, A., Escolano, E., Pascual, M., Lucas, B. y Sastre, S. (2015). Metacognición en un proceso de aprendizaje autónomo y cooperativo en el aula universitaria. Contextos Educativos, vol. 18 (95-108).

Peronard, M. (2009). Metacognición: mente y cerebro. Boletín de Filología, vol. 44 (2), 263-275.

Pozo, I. (1999). Aprendices y maestros. La nueva cultura del aprendizaje. España: Alianza Editorial.

Román, M. (2010). Pedagogía sociocognitiva como instrumento de renovación de la educación. Boletín Sociocognitivo, vol. 12, 2-7.

Saldaña, D. y Aguilera, A. (2003). La evaluación de los procesos metacognitivos: estrategias y problemáticas actuales. Estudios de Psicología, vol. 24 (2), 189-204.

Sandi-Urena, S. y Cooper. M. (2010). Evaluación y desarrollo de la metacognición en la Enseñanza de la Química. Ciencia y Tecnología, vol. 26 (1- 2), 47-57.

Solaz, J. (2010). Variables Cognitivas y Metacognitivas en la resolución de problemas de Química: Propuestas de estrategias didácticas. Química Nova, vol. 33 (6), 1403-1407.

Soto, C. (2002). Metacognición, cambio conceptual y Enseñanza de las Ciencias. Bogotá: Didáctica Magisterio.

Tesouro, M. (2005). La metacognición en la escuela: la importancia de enseñar a pensar. Educar, vol. 35,135-144.

Vásquez, A. (2015). La metacognición: una herramienta para promover un ambiente áulico inclusivo para estudiantes con discapacidad. Revista Electrónica Educare, vol. 19 (3), 1-20. 